Check for updates

Cite this: RSC Adv., 2019, 9, 36670

Received 25th August 2019

Accepted 28th October 2019

DOI: $10.1039 / c 9 r a 06690$

rsc.li/rsc-advances

\section{An amphiphilic-ligand-modified gold nanoflower probe for enhancing the stability of lateral flow immunoassays in dried distillers grains $\uparrow$}

\author{
Tongtong Ma, ${ }^{\mathrm{ab}}$ Hong Duan, ${ }^{\mathrm{ab}}$ Wenjing Zhang, ${ }^{\mathrm{ab}}$ Yanna Shao, ${ }^{\mathrm{ab}}$ Liangwen Hao, $^{\mathrm{ab}}$ \\ Xirui Chen, ${ }^{\text {ab }}$ Yuankui Leng, (iD *ac Xiaolin Huang (iD ac and Yonghua Xiong (DD *abcd
}

\begin{abstract}
An amphiphilic ligand-capped gold nanoflower (AuNF) was proposed as a novel lateral flow immunoassay (LFA) reporter for zearalenone (ZEN) detection in distillers dried grains solubles (DDGS). The amphiphilic ligand consists of a thiol-terminated hydrophobic alkane chain, a tetra (ethylene glycol) unit, and a terminal carboxyl group. The novel AuNF probe (N-AuNF-Abs) was prepared by coupling the amino group of anti-ZEN antibodies with the AuNF carboxyl group via an amido covalent linkage. For comparison, a traditional AuNF probe (Tr-AuNF-Abs) was prepared by labeling antibodies on the surface of citrate capped AuNFs via an electrostatic adsorption method. The detection performance of the two probes in LFA was systematically investigated, including the half maximal inhibitory concentration (IC $\left.\mathrm{C}_{50}\right)$, robustness and reproducibility for ZEN quantitative detection in DDGS samples, and shelf life. The $\mathrm{N}$ AuNF-Ab based LFA (N-LFA) had a lower $I_{50}$ value $\left(15.97 \mathrm{ng} \mathrm{mL} \mathrm{m}^{-1}\right.$ ) for ZEN detection in phosphate buffered saline than that of the Tr-AuNF-mAb based LFA (Tr-LFA, $31.06 \mathrm{ng} \mathrm{mL}^{-1}$ ). The IC 50 value of $\mathrm{N}$ LFA in DDGS extract was $17.46 \mathrm{ng} \mathrm{mL}^{-1}$, whereas the $\mathrm{Tr}$-LFA showed poor robustness and reproducibility in DDGS samples, resulting in a failed determination. The intra- and inter-assays of N-LFA for ZEN-spiked DDGS samples indicated that the average recoveries ranged from $93.0 \%$ to $125.9 \%$, with coefficients of variation ranging from $2.8 \%$ to $21.9 \%$. These results indicated that the N-LFA strip exhibits good robustness and an acceptable accuracy for ZEN quantitative detection in complex DDGS samples. In accelerated aging studies, N-LFA showed a longer shelf life (5 years) than that of Tr-LFA (1 year). In summary, the proposed method provided a novel strategy to prepare a super-stable probe for enhancing the detection performance of LFA for small molecular detection in complex sample matrices such as DDGS.
\end{abstract}

\section{Introduction}

${ }^{a}$ State Key Laboratory of Food Science and Technology, Nanchang University, Nanchang 330047, P. R. China. E-mail: yuankuilengxq@163.com; yhxiongchen@ 163.com

${ }^{b}$ Jiangxi-OAI Joint Research Institute, Nanchang University, Nanchang 330047, P. R. China

'School of Food Science and Technology, Nanchang University, Nanchang 330047, P. R. China

${ }^{d}$ Jiangxi Key Laboratory for Microscale Interdisciplinary Study, P. R. China

$\dagger$ Electronic supplementary information (ESI) available: Characterization of N-AuNF, N-AuNF-Abs, and Tr-AuNF, Tr-AuNF-Abs (Fig. S1 $\dagger$ ); image of physical and chemical effects of N-AuNFs and Tr-AuNFs (Fig. S2 $\dagger$ ); characterization of the stability and anti-inference ability of N-AuNF and Tr-AuNF (Fig. S3†); parameter optimization for the preparation of N-AuNF-Abs and Tr-AuNF-Abs (Fig. S4 and S5†); optimization of the parameters of the N-AuNF-Abs and Tr-AuNF-Abs based LFA strips (Table S1†); evaluation of the detection performance of N-AuNF-Abs and Tr-AuNF-Abs based LFA strips (Fig. S6-S8†); effects of some common matrix interferents on N-AuNF-Abs and Tr-AuNF-Abs based LFA strips (Fig. S9†); indirectly ELISA for anti-ZEN antibody determination (Fig. S10†); images of N-AuNF-Abs and Tr-AuNF-Abs based strips responses to PB buffer (Fig. S11†). See DOI: 10.1039/c9ra06690j
Lateral flow immunoassays (LFAs) are powerful tools for on-site screening detection in clinical diagnosis, environmental analysis, and food safety monitoring because of their simplicity, rapid results, cost-effectiveness, and user-friendliness..$^{\mathbf{1 - 5}}$ Currently, various nanomaterials, including light absorbing nanoparticles ${ }^{6-8}$ (e.g., gold nanoparticles (AuNPs), carbon nanoparticles, and colloidal selenium), light emitting nanoparticles $^{9-13}$ (e.g., dye-doped fluorescent beads, time-resolved fluorescent beads, quantum dot beads, and up-converting phosphor nanoparticles), and magnetic nanoparticles, ${ }^{\mathbf{1 4}}$ have been widely used as labels in LFAs. Among them, AuNPs are the most popular signal reporter in commercial LFAs because of their facile synthesis and modification, biocompatibility and low toxicity, low cost, and naked eye detectable results. ${ }^{15,16}$ The AuNPs traditionally used in LFAs are usually prepared via the trisodium citrate reduction method, in which trisodium citrate acts as both a reductant and a stabilizer during the AuNP synthesis. ${ }^{17}$ The resultant AuNPs typically have a core-shell 
structure, in which the atomic Au aggregate that forms the core is surrounded by an electrostatic double layer, and the citrate ligands impart a negative charge onto the surface of the AuNPs. ${ }^{18,19}$ These citrate-capped AuNPs are sensitive to the electrolyte, which can shrink the electrostatic double layers on the AuNPs and cause AuNP agglutination. Various macromolecules, such as water-soluble proteins, single-stranded DNA, carbohydrates, and polymers can adsorb onto the surface of the AuNPs through dative bonds and via electrostatic and hydrophobic forces. ${ }^{20}$ Therefore, the classical coupling protocol for antibody-labeled AuNPs (referred to from hereon as traditional AuNP and antibody conjugates, Tr-AuNP-Abs) consists of simply adding the antibodies (Abs) to the AuNP solution at a certain $\mathrm{pH}$ value. $^{21}$ To enhance the stability of the Tr-AuNP-Abs and reduce the nonspecific binding of Tr-AuNP-Abs on the nitrocellulose (NC) membrane, blocking agents, such as proteins (e.g., bovine serum albumin), sugar (e.g., sucrose and mannitose), polymers (e.g., poly(ethylene glycol), PEG), are required to block the excess binding sites on the AuNPs. ${ }^{22-24}$ However, owing to the weak binding affinity between antibodies and AuNPs, the stability of Tr-AuNP-Abs remains a serious problem when they are applied to complex sample matrices containing high levels of watersoluble proteins, metal ions, and complex carbohydrates. Therefore, using Tr-AuNP-Abs based LFAs (Tr-LFAs) for this kind of sample matrix often leads to poor reproducibility and even failed detection.

In the present study, an amphiphilic ligand (Scheme 1) was used to replace the citrate group on the AuNPs with strong Au-S bonds. $^{25}$ The amphiphilic ligand consists of a hydrophobic alkane chain, a tetra (ethylene glycol) unit, and a terminal carboxyl group. The alkane chains self-assembled into a hydrophobic layer on the surface of the AuNPs, which can prevent the ligands from detaching from the surface of the AuNPs in the presence of high concentrations of chemical compounds or complex bio-macromolecules, because it is impossible for these water-soluble compounds to penetrate the hydrophobic layer and reach the surface of the AuNPs. The tetra (ethylene glycol) unit was chosen to improve the solubility because many research groups have reported that PEG-decorated AuNPs show better biocompatibility and solubility than citrate-capped AuNPs in most sample solutions. ${ }^{2,27}$ Furthermore, the terminal carboxyl groups of the proposed ligand were used to conjugate the antibodies for the target analyte via covalent bonds. Compared with conventional spherical AuNPs, multibranched gold nanoflowers (AuNFs) exhibit stronger localized surface plasmon resonance (LSPR) absorption, better colloidal stability, and higher antibody loading capacity due to their rough surfaces and large surface-to-volume ratios. ${ }^{28-30}$ Our preliminary work demonstrated that better LFA detection performance was obtained using AuNFs than spherical AuNPs in the same format. ${ }^{25,31,32}$

Dried distillers grain with solubles (DDGS) is a byproduct of the alcoholic fermentation of cereal grains for beverages or fuel. ${ }^{33}$ DDGS has been recognized as a valuable foodstuff that provides energy, water-soluble proteins, vitamins, and minerals for animals. ${ }^{34}$ However, DDGS obtained from corn can potentially contain a higher concentration of zearalenone (ZEN) than was present in the corn prior to fermentation. ${ }^{35}$ ZEN is an estrogenic and carcinogenic mycotoxin produced by Fusarium species, in particular, $F$. graminearum, $F$. culmorum, and $F$. crookwellense. Corn is easily contaminated by Fusarium species in many parts of the world. The ingestion of ZEN by animals may cause early sexual maturity and promote the occurrence of DNA adducts and micronuclei by triggering chromosome aberrations. ${ }^{36,37}$

In this work, AuNFs modified with an amphiphilic ligand were used to conjugate anti-ZEN monoclonal antibodies via covalent coupling of the amino group of Abs to the carboxyl group of AuNF by a carbodiimide method. The proposed AuNF probe was used as a novel probe (N-AuNF-Abs) of LFA (N-LFA) for the quantitative detection of ZEN in DDGS samples. The detection performance of N-LFA in terms of its resistance to

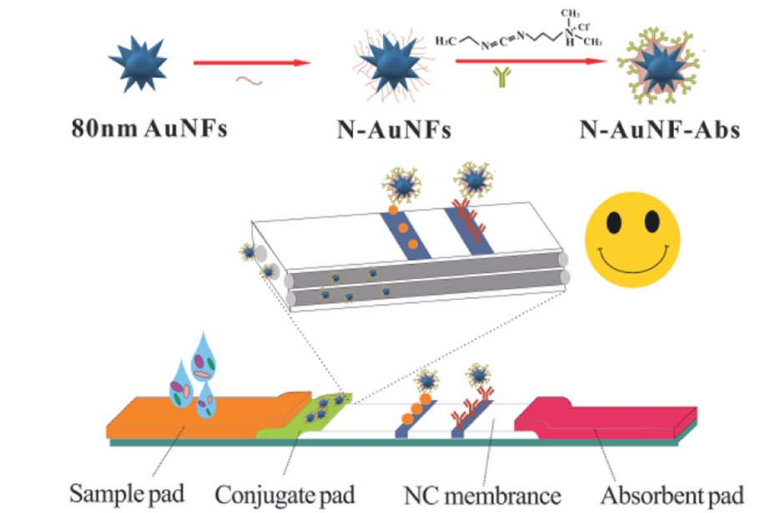

N-LFA

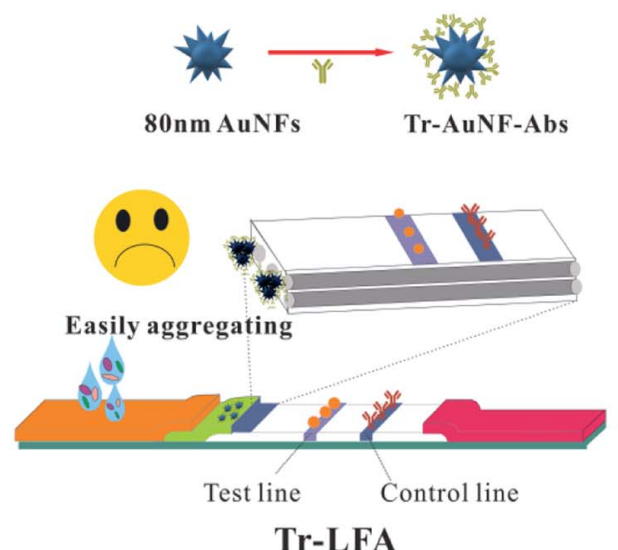

Tr-LFA
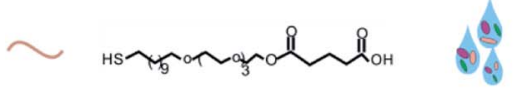

DDGS

ZEN-BSA

YI Anti-ZEN mAbs

If Goat anti-mouse IgG

Scheme 1 Schematics of the strips based on N-AuNF-Abs and Tr-AuNF-Abs for the detection of ZEN in DDGS. 
matrix interference, sensitivity and accuracy, and storage stability were investigated and compared with those of Tr-LFA. The results indicated that N-AuNF-Abs exhibited superior performance as a probe for the LFA detection of small molecular pollutants in the complex sample matrix.

\section{Model and methods}

\subsection{Materials and instruments}

$N$-(3-(Dimethylamino) propyl)- $N^{\prime}$-ethylcarbodiimidehydrochloride $(\mathrm{EDC} \cdot \mathrm{HCl})$, zearalenone $(\mathrm{ZEN})$, and bovine serum albumin (BSA) were purchased from Sigma-Aldrich Chemical (St. Louis, MO). ZEN-BSA conjugates (mole ratio of $15: 1$ ) and anti-ZEN mAbs were prepared in our laboratory. Donkey anti-mouse IgG antibodies were purchased from Beijing Zhongshan Biotechnology, Inc. (Beijing, China). The sample pad, nitrocellulose (NC) membrane, and absorbent pad were obtained from Schleicher and Schuell GmbH (Dassel, Germany). The ZEN free DDGS samples (determined by liquid chromatography-tandem mass spectrometry, LC-MS/MS) were collected from different local farms. The phosphate-buffered saline (PBS, $0.01 \mathrm{M}, \mathrm{pH}=$ 7.4) was obtained by adding $1.22 \mathrm{~g}$ of $\mathrm{K}_{2} \mathrm{HPO}_{4}, 1.36 \mathrm{~g}$ of $\mathrm{KH}_{2} \mathrm{PO}_{4}$, and $8.5 \mathrm{~g}$ of $\mathrm{NaCl}$ in $1000 \mathrm{~mL}$ of Milli-Q water and adjusted to 7.4, unless otherwise specified, before use. Phosphate buffer (PB; $0.01 \mathrm{M}, \mathrm{pH} 7.0$ ) was prepared by mixing stock solutions of $\mathrm{NaH}_{2} \mathrm{PO}_{4}$ and $\mathrm{Na}_{2} \mathrm{HPO}_{4}$. All of the other reagents were of analytical grade or better and purchased from Sinopharm Chemical Corp. (Shanghai, China).

The BioDot XYZ platform combined with a motion controller, BioJet Quanti 3000k dispenser and AirJet Quanti $3000 \mathrm{k}$ dispenser for solution dispensing was supplied by BioDot (Irvine, CA, USA).

\subsection{Preparation of citrate-stabilized AuNFs and amphiphilic-ligand-decorated AuNFs}

Citrate-capped AuNFs (Tr-AuNFs) with an average diameter of $80 \mathrm{~nm}$ were synthesized using a gold seed-mediated growth approach according to a previous report with slight modifications. ${ }^{38,39}$ Firstly, $2.7 \mathrm{~mL}$ of a $1 \%$ sodium citrate solution was added to $100 \mathrm{~mL}$ of a boiling $0.01 \%$ gold chloride trihydrate solution under constant stirring. $18 \mathrm{~nm}$ gold seeds were obtained after $10 \mathrm{~min}$ of reaction. $1.78 \mathrm{~mL}$ of the above gold seedcontaining solution was added to $100 \mathrm{~mL}$ of ultrapure water under vigorous stirring. Once the temperature reached $55{ }^{\circ} \mathrm{C}$, $1.2 \mathrm{~mL}$ of a $1 \% \mathrm{HAuCl}_{4}$ solution, $2.64 \mathrm{~mL}$ of a $1 \%$ sodium citrate solution, and $24 \mathrm{~mL}$ of $0.03 \mathrm{M}$ hydroquinone solution were immediately added in sequence. The $80 \mathrm{~nm}$ Tr-AuNFs were obtained after another $10 \mathrm{~min}$ of heating. The concentration of the resulting Tr-AuNFs was calculated based on the dosage of $18 \mathrm{~nm}$ seeds.

The amphiphilic ligand-decorated AuNFs (N-AuNFs) were obtained via exchanging the citrate group of the Tr-AuNFs with an amphiphilic ligand. ${ }^{\mathbf{4 0 , 4 1}}$ The Tr-AuNFs were mixed with an excess of the amphiphilic ligand in weakly alkaline water $(\mathrm{pH}=$ 9.0) at ambient temperature for $4 \mathrm{~h}$ under magnetic stirring. The N-AuNFs were purified via centrifugation and re-suspended in ultrapure water. The Tr-AuNFs and N-AuNFs were stored at $4{ }^{\circ} \mathrm{C}$ for further use.

\subsection{Preparation of the two kinds of AuNF probes}

$\mathrm{N}$-AuNF-Abs was synthesized by coupling the amino group of anti-ZEN mAbs with the carboxyl group on the surface of $\mathrm{N}$ AuNFs via an amide bond. ${ }^{25}$ Typically, 0.3 pmol of N-AuNFs was first mixed with $37.5 \mu \mathrm{g}$ of unpurified ascites containing $4 \%$ anti-ZEN mAbs in $15 \mathrm{~mL}$ of a $0.01 \mathrm{M}$ phosphate buffer (PB, $\mathrm{pH}=6.0$ ) at ambient temperature for $30 \mathrm{~min}$ under magnetic stirring. Three portions of a solution containing $75 \mu \mathrm{g}$ EDC were added to the solution individually, each addition was followed by $30 \mathrm{~min}$ of reaction to form amide bonds. $500 \mu \mathrm{L}$ of $10 \%$ BSA $(\mathrm{w} / \mathrm{v})$ was then added to the above mixture, and another two portions of EDC solution (75 $\mu \mathrm{g}$ ) were added separately, followed by $30 \mathrm{~min}$ of reaction each. The as-prepared N-AuNF-Abs was then purified via centrifugation and re-suspended in $0.01 \mathrm{M}$ $\mathrm{PB}$ buffer $(\mathrm{pH}=7.4)$ containing $25 \%$ sucrose and $0.1 \%$ sodium azide.

Tr-AuNF-Abs was prepared by immobilizing anti-ZEN mAbs on the surface of the Tr-AuNFs via electrostatic adsorption. $^{31,32}$ Typically, $18.9 \mu \mathrm{g}$ of unpurified ascites was added to $15 \mathrm{~mL}$ of Tr-AuNF solution (20 pM) with a pH of 7.9. After $1 \mathrm{~h}$ of incubation under gentle stirring at $25^{\circ} \mathrm{C}, 500 \mu \mathrm{L}$ of $10 \%$ BSA (w/v) was added, and the solution was incubated for another $60 \mathrm{~min}$. The as-prepared Tr-AuNF-Abs was then purified via centrifugation and resuspended in $0.01 \mathrm{M} \mathrm{PB}$ buffer $(\mathrm{pH}=7.4$ ) containing $25 \%$ sucrose and $0.1 \%$ sodium azide. Both types of AuNF probes were stored at $4{ }^{\circ} \mathrm{C}$ until further use.

\subsection{Fabrication of the ZEN-LFA}

The LFA comprised four parts: a sample pad, conjugate pad, nitrocellulose (NC) membrane, and absorbent pad. The sample pad was soaked in $0.1 \mathrm{M}$ PBS $(\mathrm{pH}=8.0)$ solution containing $1.0 \%(\mathrm{w} / \mathrm{v})$ BSA, $0.1 \%(\mathrm{w} / \mathrm{v})$ sodium azide, and $0.25 \%(\mathrm{w} / \mathrm{v})$ Tween-20, and then dried at $60{ }^{\circ} \mathrm{C}$ for $2 \mathrm{~h}$. The conjugate pad (glass fiber membrane) was doused with $0.01 \mathrm{M} \mathrm{PBS}(\mathrm{pH}=7.4)$ containing $0.2 \%$ Tween-20 and $2 \%$ sucrose and then dried at $60{ }^{\circ} \mathrm{C}$ for $4 \mathrm{~h}$. Both kinds of synthetic AuNF probes were uniformly sprayed onto separate conjugate pads using a gold spraying instrument and then vacuum-dried at $37^{\circ} \mathrm{C}$ for $90 \mathrm{~min}$. Donkey anti-mouse IgG and ZEN-BSA conjugate were spotted on the NC membrane with a density of $0.74 \mu \mathrm{L} \mathrm{cm}{ }^{-1}$ as the control (C) and test (T) lines, respectively, and then dried at $37{ }^{\circ} \mathrm{C}$ for $12 \mathrm{~h}$. The pretreated sample pad, conjugate pad, NC membrane, and absorption pad were assembled and then cut into $3.9 \mathrm{~mm} \times 60 \mathrm{~mm}$ strips. All the LFA strips were packaged in a plastic bag containing a desiccant gel and stored at ambient temperature prior to use. The typical detection procedure was as follows: $70 \mu \mathrm{L}$ of sample solution was added to the well of the sample pad. After 15 min of incubation, the optical densities of the $\mathrm{T}\left(\mathrm{OD}_{\mathrm{T}}\right)$ and $\mathrm{C}\left(\mathrm{OD}_{\mathrm{C}}\right)$ lines were recorded using an optical strip reader (Shanghai Huguo Science Instrument Co., Ltd., Shanghai, China). 


\section{Results and discussion}

\subsection{Preparation and characterization of the AuNFs}

The Tr-AuNFs were synthesized via a gold seed-mediated growth method using hydroquinone as the reductant. The morphology and size distribution of the Tr-AuNFs were characterized using a high-resolution transmission electron microscope (TEM, JEOL JEM 2100, Tokyo, Japan). The resultant AuNFs exhibited a flower-like morphology with spikes and good monodispersity with an average size of $80 \mathrm{~nm}$ (Fig. S1A $\dagger$ ). Dynamic light scattering (DLS) analysis indicated that the average hydrodynamic diameter $\left(D_{\mathrm{H}}\right)$ of the Tr-AuNFs was about $90 \mathrm{~nm}$ (Fig. S1C $\dagger$ ). In the UV-vis spectrum in Fig. S1D, $\uparrow$ the most intense surface plasmon resonance (SPR) peak of the Tr-AuNFs was located at $630 \mathrm{~nm}$. To improve the robustness of LFA, N-AuNFs, which had enhanced stability and high resistance against matrix interference, were obtained by replacing the citrate group on the surface of the Tr-AuNFs with an amphiphilic ligand via a ligand exchange process (Scheme 1). The TEM image (Fig. S1B $\dagger$ ), average $D_{\mathrm{H}}$ (Fig. S1C $\dagger$ ) and SPR peak (Fig. S1D $\dagger$ ) of the resultant $\mathrm{N}$-AuNFs were nearly the same as those of the Tr-AuNFs.

To demonstrate the enhanced stability the N-AuNFs compared to the Tr-AuNFs, the resistance of both the AuNFs to various matrix interferences, including $\mathrm{pH}$ value and the ionic strength, were evaluated. Specifically, 8 fmol of Tr-AuNFs or N-AuNFs was added to $1 \mathrm{~mL}$ samples of water with different $\mathrm{pH}$ values or concentrations of $\mathrm{NaCl}$, and the hydrodynamic size and LSPR spectra of the AuNFs were recorded after $5 \mathrm{~min}$. The results show that both the Tr-AuNFs and N-AuNFs were stable in solutions with $\mathrm{pH}$ values of 6 to 14 , and unstable in solutions with $\mathrm{pH} \leq 5$, in which they exhibited increased size (Fig. 1A) and decreased LSPR signal (Fig. 1B). As shown in Fig. 1C and D, $\mathrm{NaCl}$ concentrations of $2.5 \%$ or higher induced the aggregation of the Tr-AuNFs, resulting in a transparent solution with increased hydrodynamic size and decreased LSPR signal, while the $\mathrm{N}$-AuNFs remained stable in $5 \% \mathrm{NaCl}$ solution. The real

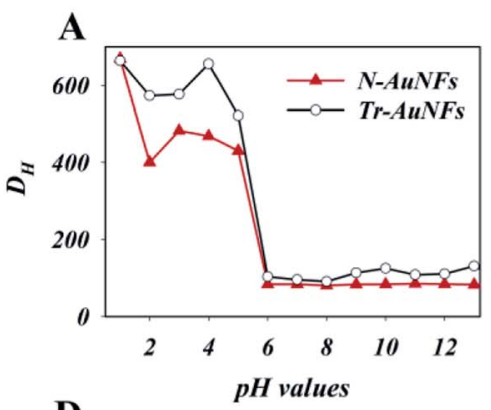

\section{B}

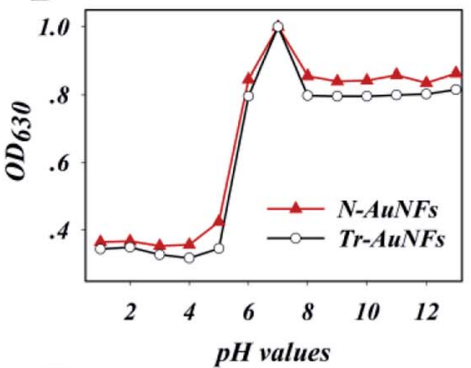

E
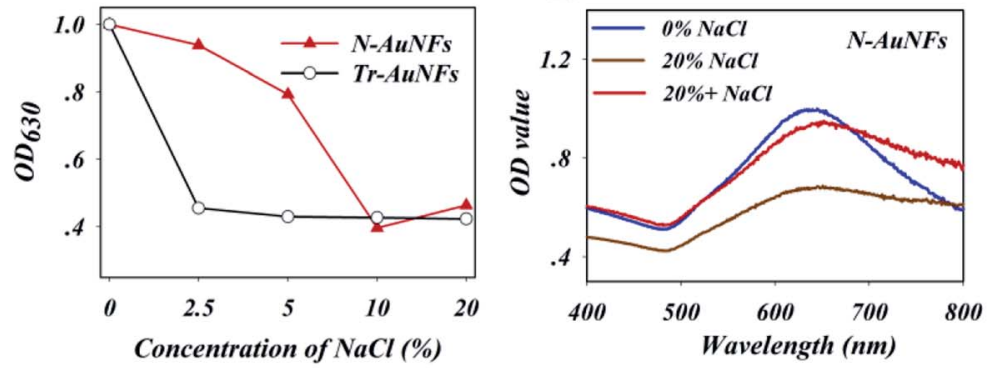

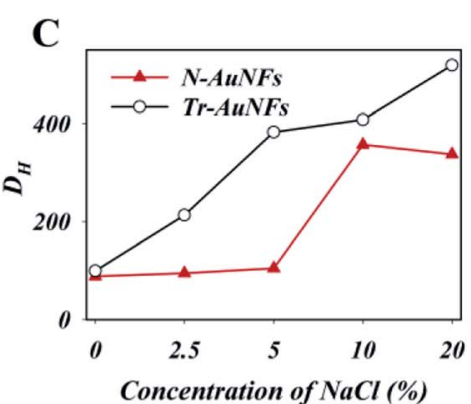

F

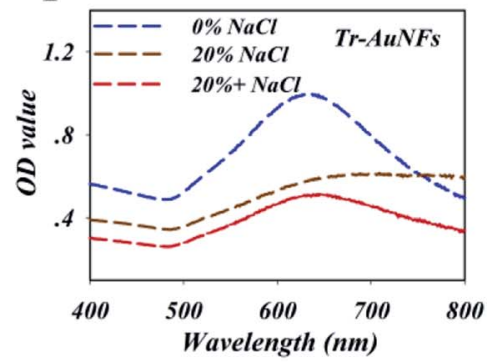

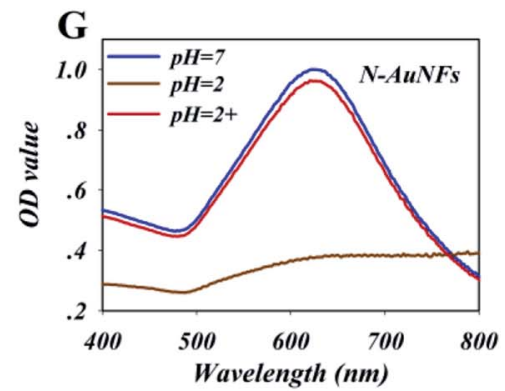

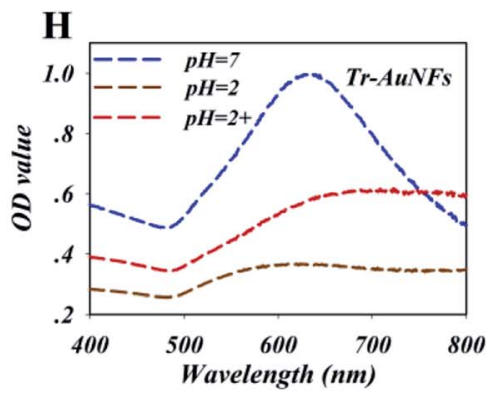

Fig. 1 The effects of the $\mathrm{pH}$ values and $\mathrm{NaCl}$ concentrations on the hydration diameters ( $\mathrm{A}$ and $\mathrm{C}$ ) and optical intensity $\left(\mathrm{OD}_{630}\right.$ value, $\mathrm{B}$ and $\left.\mathrm{D}\right)$ of the N-AuNF and Tr-AuNF solutions, respectively. UV-Vis spectra of N-AuNF (E) and Tr-AuNF (F) treated with and without $20 \%$ of $\mathrm{NaCl}$ solution (wt\%); UV-Vis spectra of N-AuNF (G) and Tr-AuNF (H) with a strongly acidic solution ( $\mathrm{pH}=2$ ); $\mathrm{N}-\mathrm{AuNF} 0 \% \mathrm{NaCl}$ and $\mathrm{Tr}-\mathrm{AuNF} 0 \% \mathrm{NaCl}$ represent that N-AuNFs and Tr-AuNFs dissolve in pure water; N-AuNF 20\% NaCl and Tr-AuNF 20\% NaCl represent that N-AuNFs and Tr-AuNFs dissolve in $20 \%$ of $\mathrm{NaCl}$ solution (wt\%); N-AuNFs $20 \%+\mathrm{NaCl}$ and Tr-AuNFs $20 \%+\mathrm{NaCl}$ represent that the aggregated N-AuNFs and aggregated Tr-AuNFs were recovered by centrifugation and then resuspended in pure water. $\mathrm{N}$-AuNFs $\mathrm{pH}=2$ and $\mathrm{Tr}$-AuNFs $\mathrm{pH}=2$ represent that N-AuNFs and TrAuNFs dissolve in a strongly acidic solution $(\mathrm{pH}=2) ; \mathrm{N}-\mathrm{AuNFs} \mathrm{pH}=2+$ and $\mathrm{Tr}$-AuNFs $\mathrm{pH}=2+$ represent that the aggregated $\mathrm{N}-\mathrm{AuNFs}$ and TrAuNFs were recovered by centrifugation and then resuspended in pure water. 
photos of both the Tr-AuNFs and N-AuNFs treated with different concentrations of $\mathrm{NaCl}$ were displayed in Fig. S2 (ESI $\dagger$ ). Moreover, unlike the Tr-AuNFs, the N-AuNF aggregates formed at a strongly acidic solution $(\mathrm{pH}=2)$ and high $\mathrm{NaCl}$ concentration $(20 \%$, wt $\%)$ could be recovered by resuspension in pure water (Fig. 1E-H and S3 $\dagger$ ). Therefore, it was concluded that the TrAuNFs irreversibly aggregated in a strongly acidic solution and high-ionic strength solution due to the destruction of the hydration layer and the detachment of the citrate ligands, while the N-AuNFs exhibited higher stability and anti-inference ability because their strong Au-S bonds and the impermeability of the hydrophobic layer by water-soluble compounds prevented the detachment of the ligands.

\subsection{Preparation and characterization of the AuNF probes}

The AuNF probes including Tr-AuNF-Abs and N-AuNF-Abs were prepared by electrostatic adsorption and covalent coupling methods, respectively. Unpurified anti-ZEN ascites were directly immobilized onto the surface of the Tr-AuNFs according to the method described in our previous report. ${ }^{17,31,32}$ To obtain the highest binding affinity between Tr-AuNF-Abs and ZEN, the coupling parameters, including the $\mathrm{pH}$ value of the Tr-AuNF solution and the dosage of ascites, were optimized. Tr-AuNFAbs obtained using different coupling conditions was used for strips with a BSA-ZEN conjugate as test line, and the optical density value at the test line $\left(\mathrm{OD}_{\mathrm{T}}\right)$ was used to evaluate the coupling efficiency and bioactivity of Tr-AuNF-Abs. The results shown in Fig. $\mathrm{S} 4 \mathrm{~A} \dagger$ indicated that the optimized $\mathrm{pH}$ value for the preparation of Tr-AuNF-Abs was 7.9, which could be achieved by adding $1 \mu \mathrm{L}$ of $0.2 \mathrm{M} \mathrm{K}_{2} \mathrm{CO}_{3}$ to $1 \mathrm{~mL}$ of Tr-AuNF solution (20 pM). Notably, the electrostatic adsorption coupling process was highly sensitive to change in the $\mathrm{pH}$ value, which would seriously decrease the reproducibility of the resulting Tr-AuNFAbs. The optimal dosage of ascites for the preparation of $\mathrm{Tr}$ AuNF-Abs was demonstrated to be $63 \mu \mathrm{g}$ for 1 pmol Tr-AuNFs (Fig. $\mathrm{S} 4 \mathrm{~B} \dagger$ ). N-AuNF-Abs was prepared by coupling the amino group of anti-ZEN mAbs to the carboxyl group of N-AuNF via the carbodiimide method. To obtain the highest affinity of N-AuNFAbs toward ZEN, the coupling parameters, including the $\mathrm{pH}$ value of the N-AuNF solution, the dosage of EDC, and the dosage of ascites were optimized, and the affinity of the resulting probes were evaluated via their performance in the strips. The results shown in Fig. S5A† demonstrate that the optimized $\mathrm{pH}$ value for the preparation of N-AuNF-Abs was 6.0. Compared to the preparation of Tr-AuNF-Abs, the covalent coupling process was much less sensitive to changes in the $\mathrm{pH}$ value, and thus would enable highly robust preparation of $\mathrm{N}$ AuNF-Abs. Three or two portions of EDC solution were added regularly during the ascite immobilization process and BSA blocking process; each portion contained $5 \mu \mathrm{g}$ EDC per $1 \mathrm{~mL}$ of $\mathrm{N}$-AuNF solution (20 pM), according to the results shown in Fig. S5B. $\uparrow$ The optimized dosage of ascites for the preparation of

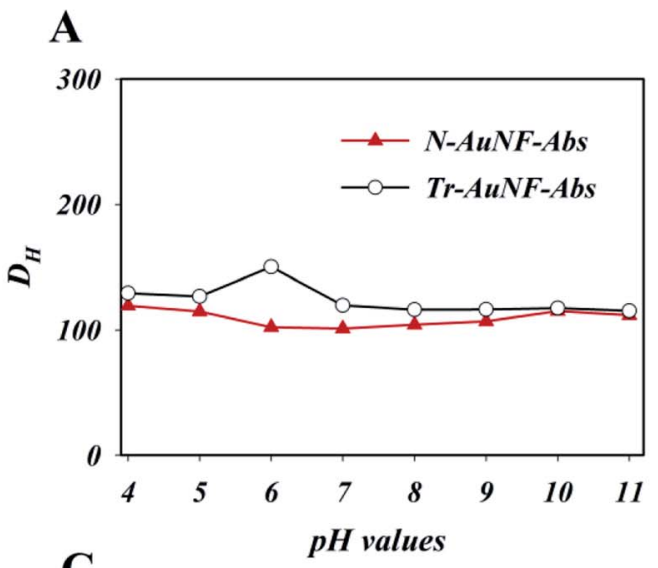

B
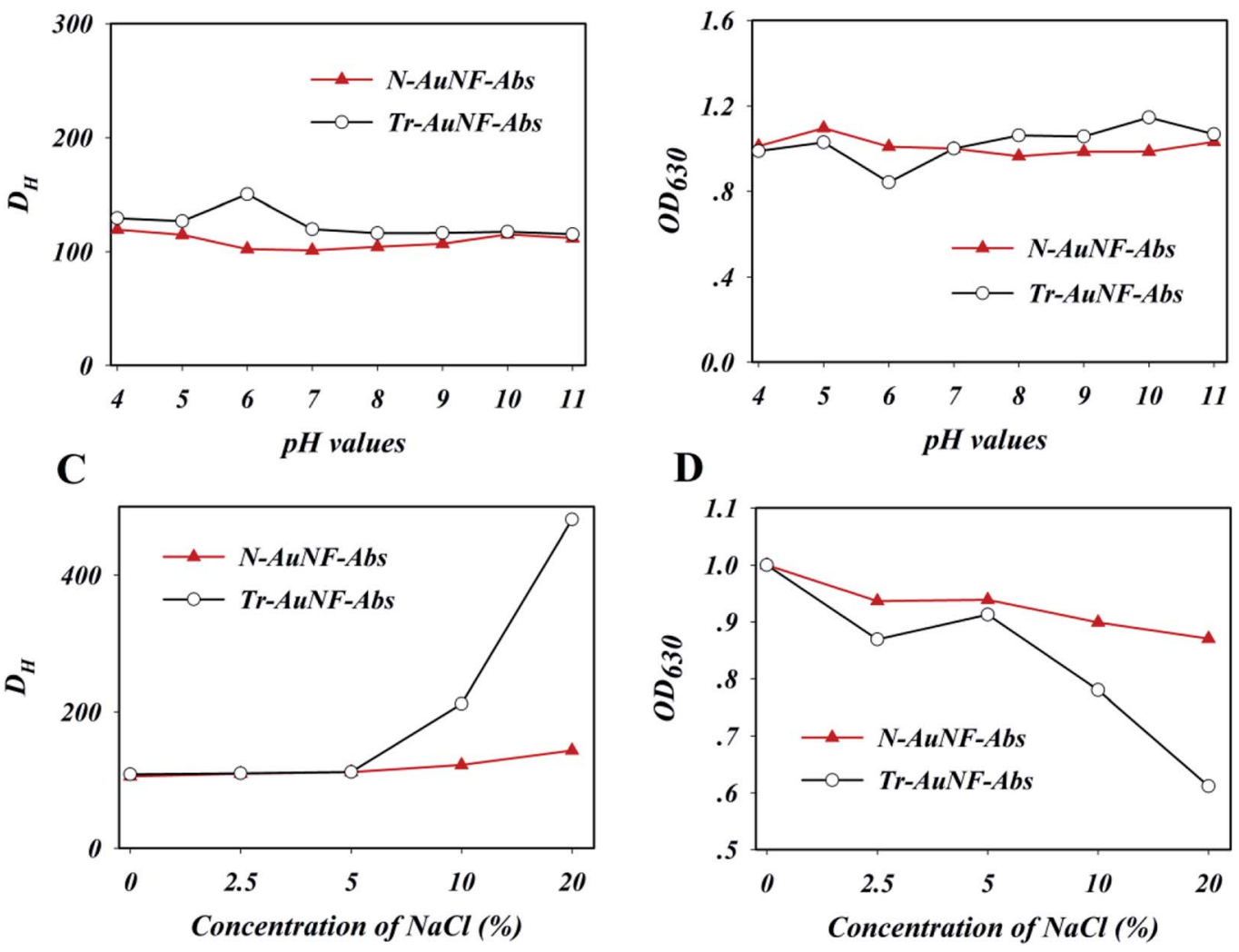

Fig. 2 The effects of the $\mathrm{pH}$ values and $\mathrm{NaCl}$ concentrations on the hydration diameters $(\mathrm{A}$ and $\mathrm{C})$ and optical intensity $\left(O \mathrm{D}_{630}\right.$ value, $\mathrm{B}$ and $\left.\mathrm{D}\right)$ of the N-AuNF-Abs and Tr-AuNF-Abs. $\mathrm{OD}_{630}$ values were normalized by dividing them by the $\mathrm{OD}_{630}$ value at $\mathrm{pH}=7.0 \mathrm{or} 0 \mathrm{wt} \% \mathrm{NaCl}$. 
N-AuNF-Abs was demonstrated to be $125 \mu \mathrm{g}$ for 1 pmol N-AuNFs (Fig. S5C $\dagger$ ), which was higher than the dosage used in the preparation of Tr-AuNF-Abs due to the relatively lower coupling efficiency. The average $D_{\mathrm{H}}$ values of the resultant Tr-AuNF-Abs and N-AuNF-Abs were slightly higher than those of the TrAuNFs and N-AuNFs, respectively (Fig. S1C $\dagger$ ). Slight red shifts in the SPR peaks of Tr-AuNF-Abs and N-AuNF-Abs were observed after immobilization of the antibody (Fig. S1D†).

The resistance of the resulting Tr-AuNF-Abs and N-AuNF-Abs complexes against different $\mathrm{pH}$ values and ionic strengths (simulated by the concentration of $\mathrm{NaCl}$ ) were evaluated. Specifically, to investigate the stability of the AuNF probes, 5 fmol of Tr-AuNF-Abs or N-AuNF-Abs was added to $1 \mathrm{~mL}$ of $0.01 \mathrm{M}$ PB with different $\mathrm{pH}$ values or $\mathrm{NaCl}$ contents, and the hydrodynamic sizes and LSPR spectra of the AuNFs were recorded after $5 \mathrm{~min}$. As demonstrated in Fig. $2 \mathrm{~A}$ and $\mathrm{B}$, both $\mathrm{N}$ AuNF-Abs and Tr-AuNF-Abs showed high colloidal stability in the presence of a wide range $\mathrm{pH}$ values (4-11). The results in Fig. 2C demonstrate that Tr-AuNF-Abs in solutions with a $\mathrm{NaCl}$ content greater than $5 \mathrm{wt} \%$ exhibited a larger $D_{\mathrm{H}}$ and lower SPR signal than Tr-AuNF-Abs in a $0.01 \mathrm{M}$ PB buffer (0 wt\%), indicating the aggregation of Tr-AuNF-Abs; whereas the N-AuNF-Abs showed a negligible change in $D_{\mathrm{H}}$ and SPR signal even $\mathrm{NaCl}$ concentration up to $20 \mathrm{wt} \%$ (Fig. 2D), indicating that N-AuNFAbs has a higher resistance against high ionic strength. Notably, compared with the corresponding AuNFs, both the AuNF probes showed higher resistance against high ionic strength or acidic conditions, which may have been due to the shielding effect of the proteins on their surfaces.

\subsection{Development of AuNF probe-based ZEN-LFA}

The two AuNF probes were then used to produce ZEN-LFA strips. The density of ZEN-BSA on the test line and the density of the AuNF probes on the conjugated pad were first optimized via an orthogonal experiment, in which the inhibition rate of a ZEN-spiked PB sample $\left(5 \mathrm{ng} \mathrm{mL}{ }^{-1}\right)$ was used as the evaluation criterion. The inhibition rate was calculated as $(1-$ $\left.B / B_{0}\right) \times 100$, where $B_{0}$ and $B$ represent the ratio of the OD values of the $\mathrm{T}$ line and $\mathrm{C}$ line $(\mathrm{T} / \mathrm{C})$ obtained from detecting the negative sample and a ZEN-positive sample, respectively. As shown in Table $\mathrm{S} 1, \uparrow$ for the N-AuNF-Abs-based test strip, the optimized densities were $1.48 \mu \mathrm{g} \mathrm{cm} \mathrm{cm}^{-1}$ ZEN-BSA and 1 fmol $\mathrm{cm}^{-1} \mathrm{~N}$-AuNF-Abs, while for the Tr-AuNF-Abs based test strip, $0.37 \mu \mathrm{g} \mathrm{cm}^{-1}$ ZEN-BSA and $3 \mathrm{fmol} \mathrm{cm}^{-1}$ Tr-AuNF-Abs were optimum. Moreover, $15 \mathrm{~min}$ was selected as the reaction time for further experiments based on the results of immunological kinetics analysis (Fig. S6†). A high methanol content was necessary to achieve a high extraction recovery from the ZENpolluted samples due to the strong hydrophobicity of ZEN, however, the presence of methanol in the sample solution can influence the antigen-antibody interactions. Thus, 0.01 M PB buffer solutions $(\mathrm{pH}=7.0)$ with different methanol contents $(0-$ $40 \%$ ) were tested with the two ZEN-LFA strips to evaluate the effect of methanol content. As shown in Fig. S7, $\uparrow$ increasing the methanol content had an obvious effect on the signals (i.e., $\mathrm{OD}_{\mathrm{T}}$, and $\mathrm{T} / \mathrm{C}$ values) of both strips. Thus, $5 \%$ was chosen as the optimum methanol content for subsequent experiments, as this concentration had little effect on the two ZEN-LFA strips.

The stability of the as-prepared ZEN-LFA strips during longterm storage was explored using accelerated aging studies. Specifically, the strips were aged at $60{ }^{\circ} \mathrm{C}$ over a period of 45 days. Negative samples (0.01 M PB buffer, $\mathrm{pH}=7.0)$ were added to the test strips regularly, and the $\mathrm{OD}_{\mathrm{T}}$ and $\mathrm{OD}_{\mathrm{C}}$ values were recorded. As shown in Fig. $3 \mathrm{~A}$, the resulting $\mathrm{OD}_{\mathrm{T}}$ and $\mathrm{OD}_{\mathrm{C}}$ values of the N-AuNF-Abs-based test trips decreased over the first six days of accelerated storage, then exhibited a plateau from day 7 to day 45 with a stabilized $\mathrm{T} / \mathrm{C}$ value. In contrast, the $\mathrm{OD}_{\mathrm{T}}$ and $\mathrm{OD}_{\mathrm{C}}$ values of the Tr-AuNF-Abs-based test trips remained constant from day 1 to day 12 with a stable T/C value (Fig. 3B). Using the Arrhenius equation, ${ }^{\mathbf{4 2 , 4 3}}$ it was preliminarily estimated that the shelf lives of the N-AuNF-Abs based test strips and TrAuNF-Abs based test strips at ambient temperature would be approximately 5 years and 1 year. The N-AuNF-Abs and Tr-AuNFAbs based test strips were aged at $60{ }^{\circ} \mathrm{C}$ for 6 days and $24 \mathrm{~h}$, respectively, after preparation, and then stored in a drying tower at ambient temperature for further usage.

The analytical performance of the two ZEN-LFA strips in the detection of ZEN-spiked PB buffer $(0.01 \mathrm{M}, \mathrm{pH}=7.0,5 \%$ methanol) samples was then evaluated. Calibration curves (Fig. 3C and D) were obtained by plotting the resulting $B / B_{0}$ values against the logarithm of the ZEN concentrations (0-1.25 $\mu \mathrm{g} \mathrm{mL}^{-1}$ ), where $B$ and $B_{0}$ were $\mathrm{T} / \mathrm{C}$ values obtained from detecting positive samples and negative samples, respectively. Then corresponding linear detection ranges, along with their regression equations and detection sensitivities (half-maximum inhibitory concentration, $\mathrm{IC}_{50}$ ), are also shown in Fig. $3 \mathrm{C}$ and $\mathrm{D}$. N-LFA showed sensitive detection, with an $\mathrm{IC}_{50}$ of $15.97 \mathrm{ng}$ $\mathrm{mL}^{-1}$ and a limit of detection (LOD, $\mathrm{IC}_{10}$ ) of $2.31 \mathrm{ng} \mathrm{mL} \mathrm{m}^{-1}$. This $\mathrm{IC}_{50}$ value was approximately half that of Tr-LFA $\left(\mathrm{IC}_{50}=31.06\right.$ $\mathrm{ng} \mathrm{mL}^{-1}$, LOD $\left.=3.59 \mathrm{ng} \mathrm{mL}^{-1}\right)$. Samples spiked with high concentrations (800 $\mathrm{ng} \mathrm{mL}^{-1}$ ) of other mycotoxins (fumonisin $\mathrm{B}_{1}$, aflatoxin $\mathrm{B}_{1}$, aflatoxin $\mathrm{B}_{2}$, deoxynivalenol, aflatoxin $\mathrm{G}_{1}$, citrinin, and ochratoxin A) were used to evaluate the specificity of the two test strips. The results in Fig. S8 $\uparrow$ show that both ZENLFA strips could specifically detect ZEN.

\subsection{Validation of the ZEN-LFA strips for ZEN detection in DDGS}

The two ZEN-LFA strips were then used to detect ZEN in a DDGS sample. The primary DDGS extract solution was prepared by extracting a $1 \mathrm{~g}$ DDGS sample with $5 \mathrm{~mL}$ of the extraction solvent (40\% v/v 0.01 M PB buffer, 60\% methanol, $\mathrm{pH}=7.0$ ) for $30 \mathrm{~min}$. The solution was then diluted 12-fold with $0.01 \mathrm{M} \mathrm{PB}$ buffer $(\mathrm{pH}=7.0)$. Calibration curves (Fig. 3E and $\mathrm{F}$ ) of the two ZEN-LFA strips for the detection of ZEN were prepared using spiked DDGS extract solution samples prepared from ZENnegative DDGS samples. The N-LFA strips exhibited nearly the same sensitivity in the DDGS samples as in the PB buffer, with an $\mathrm{IC}_{50}$ of $17.46 \mathrm{ng} \mathrm{mL} \mathrm{m}^{-1}$ and LOD of $2.84 \mathrm{ng} \mathrm{mL}^{-1}$, indicating their high anti-interference ability (Fig. 3E). In contrast, the tests of ZEN-spiked DDGS extraction solutions using Tr-LFA strips yielded less robust data with much greater standard 
A

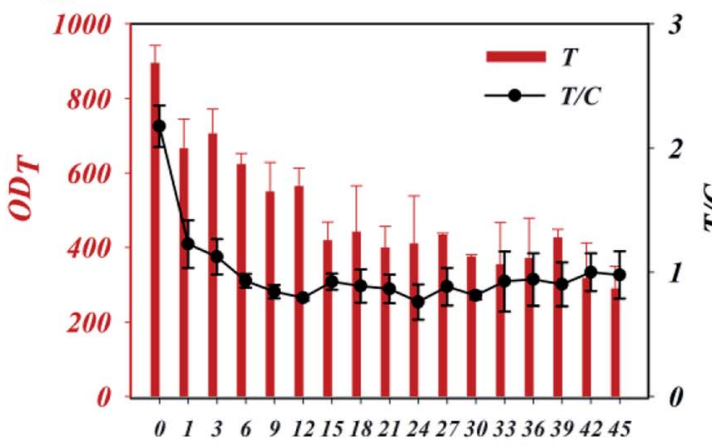

C

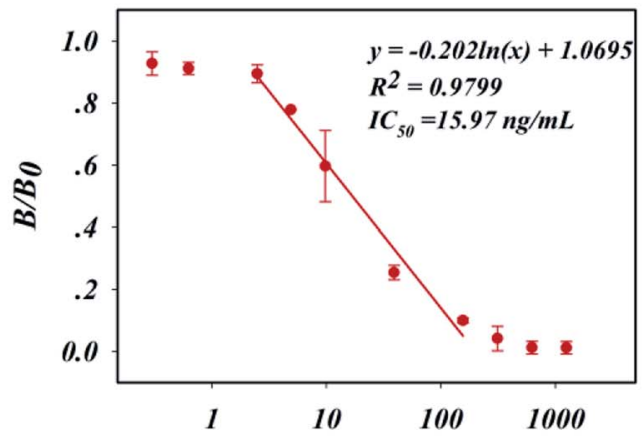

$\mathbf{E}$

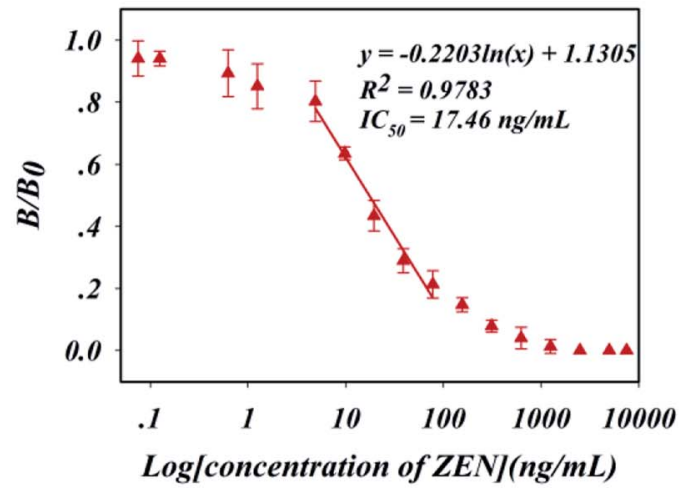

B

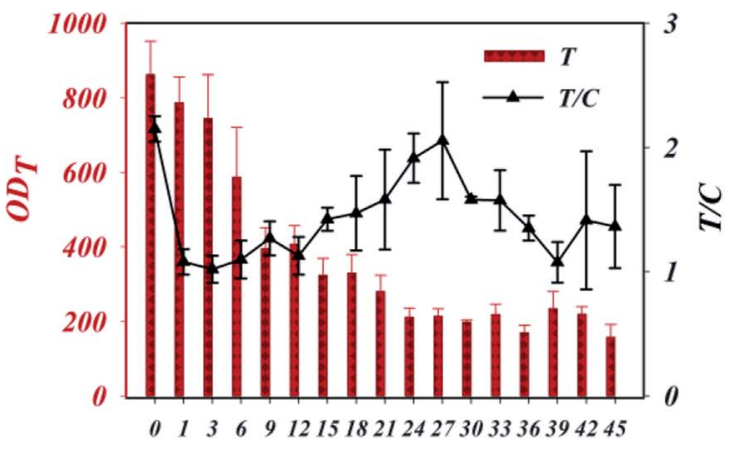

Time (day)

D

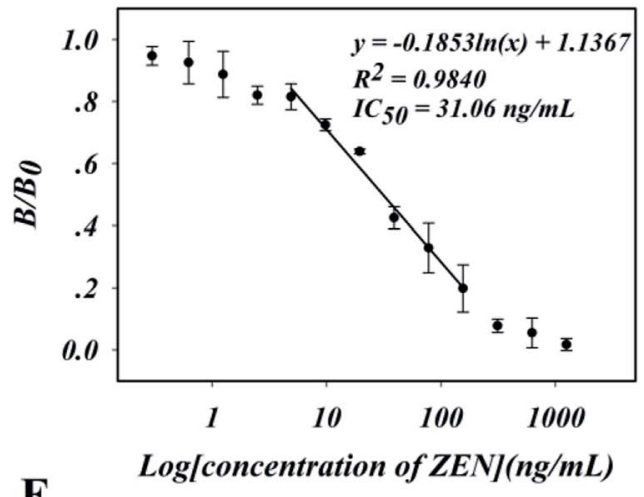

F

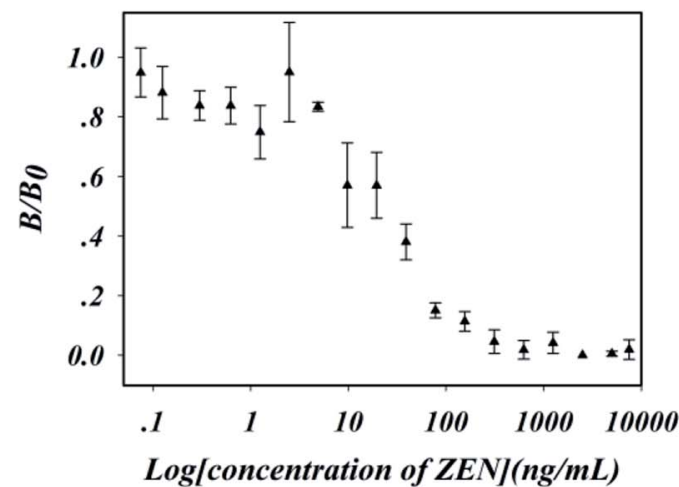

Fig. 3 Changes in the signals $\left(O D_{T}\right.$, and T/C values) of the (A) N-AuNF-Abs and (B) Tr-AuNF-Abs based ZEN-LFA strips in response to negative samples (0.01 M PB buffer, $\mathrm{pH}=7.0$ ) during a 45 day aging period at $60^{\circ} \mathrm{C}$. Calibration curves of the (C) N-AuNF-Abs- and (D) Tr-AuNF-Absbased ZEN-LFA strips for detecting ZEN-spiked PB $(0.01 \mathrm{M}, \mathrm{pH}=7.0)$ samples. Calibration curves of the (E) N-AuNF-Abs and (F) Tr-AuNF-Abs based ZEN-LFA strips for detecting ZEN-spiked DDGS extract (5\% methanol) samples.

deviations; the $\mathrm{IC}_{50}$ could not be determined from the obtained calibration curve, which contained several discontinuities (Fig. 3F). Intra-day and inter-day assays were then conducted to evaluate the accuracy and reproducibility of the N-LFA strips using ZEN-spiked DDGS extract solutions with different concentrations $\left(4.9,19.5\right.$, and $\left.78 \mathrm{ng} \mathrm{mL}^{-1}\right)$. Intra-day assays were carried out within 1 day with five replicates at each spiking level, whereas inter-day assays were conducted on three consecutive days with five replicates at each spiking level. The average recoveries for the intra-day assays ranged from $98.5 \%$ to $124.3 \%$ with coefficients of variation (CVs) ranging from $2.8 \%$ to $14.5 \%$, while the average inter-day recoveries ranged from
93.0\% to $125.9 \%$ with CVs ranging from $7.0 \%$ to $21.9 \%$ (Table 1). The above results demonstrated that the N-AuNF-Absbased strip was superior to the Tr-AuNF-Abs-based strip for the detection of ZEN in DDGS, with higher detection sensitivity, greater robustness, higher reproducibility, and better accuracy.

The mechanism by which the matrix interfered with analytical performance of the Tr-LFA strips was then explored. In theory, the negative impact of the sample matrix on the ZENLFA strips may have been due to its influence on antigenantibody interactions or decreased stability of the AuNF probes. First, the effects of some common sources of matrix interference, including the $\mathrm{pH}$ and ionic strength, on the two ZEN-LFA 
Table 1 Precision and accuracy evaluation of the N-AuNF-Abs-based strips with ZEN-spiked DDGS extract (5\% methanol)

\begin{tabular}{|c|c|c|c|c|c|c|}
\hline $\begin{array}{l}\text { Spiked concentration } \\
\text { (ng } \mathrm{mL}^{-1} \text { ) }\end{array}$ & \multicolumn{3}{|l|}{ Intra-assay } & \multicolumn{3}{|l|}{ Inter-assay $^{a}$} \\
\hline 4.9 & 4.8 & 98.5 & 7.5 & 4.6 & 93.0 & 16.9 \\
\hline 19.5 & 27.6 & 111.9 & 14.5 & 26.4 & 125.9 & 21.9 \\
\hline 78.0 & 97.0 & 124.3 & 2.8 & 120.5 & 120.5 & 7.0 \\
\hline
\end{tabular}

${ }^{a}$ Assay was completed every 1 day for 3 days continuously. ${ }^{b}$ Mean value of five replicates at each spiked concentration.

strips were evaluated. Specifically, negative samples (0.01 M PB buffer) containing different matrix interferents were tested using the two ZEN-LFA strips. The results in Fig. S9A and $\mathrm{B} \dagger$ demonstrate that the two ZEN-LFA strips showed similar changes in their $\mathrm{OD}_{\mathrm{T}}$ and $\mathrm{OD}_{\mathrm{C}}$ responses with changing $\mathrm{pH}$ values (4-11), which may account for the influence of the $\mathrm{pH}$ value on the antigen-antibody interaction on the $\mathrm{T}$ line and $\mathrm{C}$ line. As shown in Fig. S9C and $\mathrm{D}, \dagger$ the presence of $0-2.5 \mathrm{wt} \%$ $\mathrm{NaCl}$ in the samples $(0.01 \mathrm{M} \mathrm{PB}, \mathrm{pH}=7.0)$ had little effect on either of the ZEN-LFA strips. Thus, it was concluded that the effect of common matrix interferents, including the $\mathrm{pH}$ value and ionic strength, could account for the changes in antigenantibody interactions, and no obvious differences in the responses of the two ZEN-LFA strips to these interferents were observed.

Therefore, the high robustness of N-LFA strips for the detection of ZEN in DDGS may have been the result of the high stability and anti-interference ability of N-AuNF-Abs, which were provided by the amphiphilic ligand and the covalent coupling of the antibodies, while the lower stability of Tr-AuNFAbs could be accounted for by the decreased colloidal stability or the detachment of the antibodies. To test these assumptions, an ELISA-based experiment to quantify the detachment of the antibodies was conducted. Specifically, three different solutions ( 0.01 M PBS containing $20 \%, 40 \%$, or $60 \%$ methanol, $\mathrm{pH}=7.4$ ) were used to extract ZEN-negative DDGS, and then three different DDGS extractions (5\% methanol) containing different matrix interferents were obtained by dilution with PBS buffer. The two AuNF probes (12.5 fmol) were separately incubated with $350 \mu \mathrm{L}$ of each of the three DDGS extractions or PBS buffer for $30 \mathrm{~min}$, followed by a centrifugation process. The concentrations of anti-ZEN antibodies in the eight resulting supernatants were detected using an established conventional ELISA technique, in which BSA-ZEN conjugate was used as the capture
A

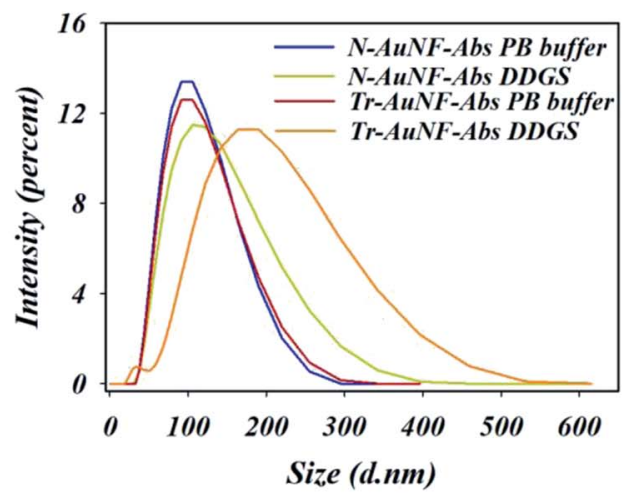

C

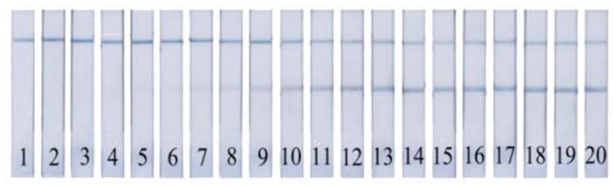

ZEN-spiked DDGS extract (N-LFA)
B

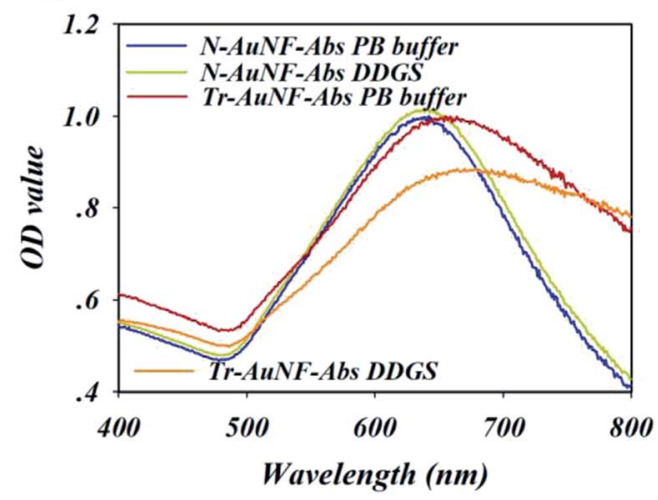

D

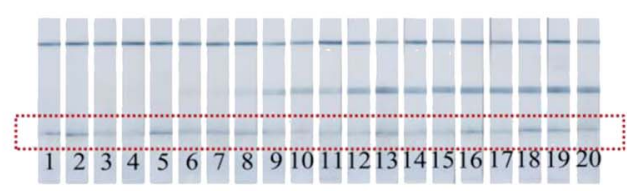

ZEN-spiked DDGS extract (Tr-LFA)

Fig. 4 (A) DLS analysis and (B) UV-Vis spectra of N-AuNF-Abs and Tr-AuNF-Abs suspended in DDGS extractions ( $5 \%$ methanol) and PB buffer (pH =7.0), recorded 5 min after the addition of the AuNFs. Images of test strip responses to different samples, (C) N-AuNF-Abs and (D) Tr-AuNF-Abs based strips with ZEN-spiked DDGS extract (5\% methanol). Samples 1-20 of each group were spiked with different concentrations of ZEN from 0 to $7.5 \mu \mathrm{g} \mathrm{mL}^{-1}$ (the corresponding concentrations of $1-20$ are $7.5 \mu \mathrm{g} \mathrm{mL}^{-1}, 5.0 \mu \mathrm{g} \mathrm{mL}^{-1}, 2.5 \mu \mathrm{g} \mathrm{m}^{-1}, 1.25 \mu \mathrm{gL}^{-1}, 625 \mathrm{ng} \mathrm{mL}^{-1}, 313 \mathrm{ng} \mathrm{mL}^{-1}$, $156 \mathrm{ng} \mathrm{mL}^{-1}, 78 \mathrm{ng} \mathrm{mL}^{-1}, 39 \mathrm{ng} \mathrm{mL}^{-1}, 20 \mathrm{ng} \mathrm{mL}^{-1}, 9.8 \mathrm{ng} \mathrm{mL}^{-1}, 4.9 \mathrm{ng} \mathrm{mL}^{-1}, 2.45 \mathrm{ng} \mathrm{mL}^{-1}, 1.225 \mathrm{ng} \mathrm{mL}^{-1}, 0.613 \mathrm{ng} \mathrm{mL}^{-1}, 0.3 \mathrm{ng} \mathrm{mL}^{-1}, 0.15 \mathrm{ng}^{-1}$ $\mathrm{mL}^{-1}, 0.075 \mathrm{ng} \mathrm{mL}^{-1}, 0.038 \mathrm{ng} \mathrm{mL}^{-1}$, and 0 , respectively). 
probe and horseradish peroxidase (HRP)-IgG conjugate was used as the reporter (Fig. S10 $\dagger$ ). The results showed the negligible content of antibodies was found in the supernatant, indicating that the detachment of the antibody was not the reason for the low stability of Tr-AuNF-Abs in DDGS. However, Tr-AuNF-Abs exhibited the remarkably increased $D_{\mathrm{H}}$ and decreased SPR signal in the DDGS extract, while the $D_{\mathrm{H}}$ and SPR signal of N-AuNF-Abs were almost the same in both PBS and DDGS extract (Fig. 4A and B). In addition, compared with NAuNF-Abs, the significantly clogging of the AuNF probes has occurred on the Tr-AuNF-Abs based strips when the ZEN-spiked DDGS samples were used (Fig. 4C, D and S11 $\dagger$ ). Therefore, it can be inferred that aggregation of Tr-AuNF-Abs induced by matrix interference was the primary reason for the low robustness of the Tr-AuNF-Abs-based strips. We speculated that possible mechanism for interferent-induced aggregation of the Tr-AuNFAbs could involve the replacement of the BSA on the surfaces of the AuNFs by interferents in the DDGS. For instance, BSA could be replaced by liposoluble substances or substances with polyvalent bonding ability towards AuNFs (e.g., proteins with multiple thiol groups available), while for N-AuNF-Abs, such replacement could be avoided.

\section{Conclusions}

Herein, a novel AuNF probe (N-AuNF-Abs) with enhanced stability was prepared by immobilizing antibodies onto AuNFs capped with amphiphilic ligands terminated by thiol and carboxyl groups via covalent coupling. A traditional AuNF probe (Tr-AuNF-Abs) was also prepared by modifying the antibodies onto citrate-capped AuNFs via electrostatic adsorption and used as a comparison. The Tr-AuNF-Abs probe showed interferenceinduced aggregation, as a result, the ZEN-LFA based on this probe was not robust enough for the analysis of DDGS samples. The as-prepared N-AuNF-Abs probe showed higher stability and anti-interference ability, with no interference-induced aggregation. The N-AuNF-Abs based LFA showed high robustness in the detection of ZEN in DDGS samples. Under optimal conditions, the proposed strips exhibited nearly the same sensitivity, with an $\mathrm{IC}_{50}$ of $17.46 \mathrm{ng} \mathrm{mL} \mathrm{m}^{-1}$ and LOD of $2.84 \mathrm{ng} \mathrm{mL}^{-1}$, for detection in DDGS samples and in PB buffer. The average recoveries for the detection of ZEN-spiked DDGS samples ranged from $90.0 \%$ to $130.0 \%$ with a CV of less than $22 \%$, indicating the high accuracy and excellent reproducibility of the proposed ZEN-LFA strips. In conclusion, the N-AuNF-Abs-based LFA achieved highly sensitive and robust detection of ZEN in DDGS samples, and also showed promising potential for the determination of other pollutants in complex sample matrices.

\section{Conflicts of interest}

There are no conflicts to declare.

\section{Acknowledgements}

This work was supported by a grant from the National Key Research and Development Program of China
(2018YFC1602203, 2018YFC1602505, and 2018YFC1602202), National Natural Science Foundation of China (31760485), and NCUIIF (Interdisciplinary Innovation Fund of Natural Science, Nanchang University) Project No. 9166-27060003-ZD01.

\section{References}

1 X. Huang, Z. P. Aguilar, H. Xu, W. Lai and Y. Xiong, Biosens. Bioelectron., 2016, 75, 166-180.

2 M. Sajid, A.-N. Kawde and M. Daud, J. Saudi Chem. Soc., 2015, 19, 689-705.

3 D. Quesada-González, A. Sena-Torralba, W. P. Wicaksono, A. de la Escosura-Muñiz, T. A. Ivandini and A. Merkoçi, Biosens. Bioelectron., 2019, 132, 132-135.

4 H. Duan, X. Huang, Y. Shao, L. Zheng, L. Guo and Y. Xiong, Anal. Chem., 2017, 89, 7062-7068.

5 K. G. Shah, V. Singh, P. C. Kauffman, K. Abe and P. Yager, Anal. Chem., 2018, 90, 6967-6974.

6 Z. Shen, W. Baker, H. Ye and Y. Li, Nanoscale, 2019, 11, 73717385.

7 C. Suárez-Pantaleón, J. Wichers, A. Abad-Somovilla, A. van Amerongen and A. Abad-Fuentes, Biosens. Bioelectron., 2013, 42, 170-176.

8 L. Chen, Z. Deng, C. Zhong, Y. Zhou and Y. Bai, Cryst. Growth Des., 2016, 16, 2581-2589.

9 N. A. Taranova, A. N. Berlina, A. V. Zherdev and B. B. Dzantiev, Biosens. Bioelectron., 2015, 63, 255-261.

10 B. Huang, H. Yu, J. Bao, M. Zhang, W. L. Green and S.-Y. Wu, Biomarker Insights, 2018, 13, 1177271918757484.

11 Y. Shao, H. Duan, L. Guo, Y. Leng, W. Lai and Y. Xiong, Anal. Chim. Acta, 2018, 1025, 163-171.

12 Z. Liang, X. Wang, W. Zhu, P. Zhang, Y. Yang, C. Sun, J. Zhang, X. Wang, Z. Xu, Y. Zhao, R. Yang, S. Zhao and L. Zhou, Mater. Interfaces, 2017, 9, 3497-3504.

13 D. Lou, L. Fan, Y. Cui, Y. Zhu, N. Gu and Y. Zhang, Anal. Chem., 2018, 90, 6502-6508.

14 L. Guo, Y. Shao, H. Duan, W. Ma, Y. Leng, X. Huang and Y. Xiong, Anal. Chem., 2019, 91, 4727-4734.

15 Z. Gao, H. Ye, D. Tang, J. Tao, S. Habibi, A. Minerick, D. Tang and X. Xia, Nano Lett., 2017, 17, 5572-5579.

16 T. Robson, D. S. H. Shah, A. S. Solovyova and J. H. Lakey, ACS Appl. Nano Mater., 2018, 1, 3590-3599.

17 J. Li, H. Duan, P. Xu, X. Huang and Y. Xiong, RSC Adv., 2016, 6, 26178-26185.

18 S. K. Ghosh and T. Pal, Chem. Rev., 2007, 107, 4797-4862.

19 C. K. K. Choi, Y. T. E. Chiu, X. Zhuo, Y. Liu, C. Y. Pak, X. Liu, Y.-L. S. Tse, J. Wang and C. H. J. Choi, ACS Nano, 2019, 13, 5864-5884.

20 J. Gao, X. Huang, H. Liu, F. Zan and J. Ren, Langmuir, 2012, 28, 4464-4471.

21 L.-K. Lin, A. Uzunoglu and L. A. Stanciu, Small, 2018, 14, 1702828.

22 K. Rahme, J. Guo, J. D. Holmes and C. M. O'Driscoll, Colloids Surf., B, 2015, 135, 604-612.

23 C. Couto, R. Vitorino and A. L. Daniel-da-Silva, Crit. Rev. Biotechnol., 2017, 37, 238-250.

24 Z. He, J. Liu and L. Du, Nanoscale, 2014, 6, 9017-9024. 
Paper

View Article Online

RSC Advances

25 W. Zhang, H. Duan, R. Chen, T. Ma, L. Zeng, Y. Leng and Y. Xiong, Talanta, 2019, 194, 604-610.

26 J. Manson, D. Kumar, B. J. Meenan and D. Dixon, Gold Bull., 2011, 44, 99-105.

27 Y. Chen, Y. Xianyu and X. Jiang, Acc. Chem. Res., 2017, 50, 310-319.

28 A. Guerrero-Martínez, S. Barbosa, I. Pastoriza-Santos and L. M. Liz-Marzán, Curr. Opin. Colloid Interface Sci., 2011, 16, 118-127.

29 F. Liebig, S. Moreno, A. F. Thünemann, A. Temme, D. Appelhans and J. Koetz, Colloids Surf., B, 2018, 167, 560-567.

30 Y. Imura, S. Koizumi, R. Akiyama, C. Morita-Imura and T. Kawai, Langmuir, 2017, 33, 4313-4318.

31 Y. Ji, M. Ren, Y. Li, Z. Huang, M. Shu, H. Yang, Y. Xiong and Y. Xu, Talanta, 2015, 142, 206-212.

32 P. Xu, J. Li, X. Huang, H. Duan, Y. Ji and Y. Xiong, Anal. Methods, 2016, 8, 3316-3324.

33 D. J. Johnston and R. A. Moreau, Bioresour. Technol., 2017, 226, 118-124.
34 H.-J. Yang, J.-H. Lee, M. Won and K. B. Song, Food Chem., 2016, 196, 174-179.

35 K. Bi, W. Zhang, Z. Xiao and D. Zhang, AMB Express, 2018, 8, 194.

36 P. Pidenko, H. Zhang, P. Lenain, I. Goryacheva, S. De Saeger and N. Beloglazova, Anal. Chim. Acta, 2018, 1040, 99-104.

37 S. Wu, L. Liu, N. Duan, Q. Li, Y. Zhou and Z. Wang, J. Agric. Food Chem., 2018, 66, 1949-1954.

38 R. P. M. Höller, M. Dulle, S. Thomä, M. Mayer, A. M. Steiner, S. Förster, A. Fery, C. Kuttner and M. Chanana, ACS Nano, 2016, 10, 5740-5750.

39 G. Frens, Nat. Phys. Sci., 1973, 241, 20-22.

40 Y. Zhou, X. Huang, W. Zhang, Y. Ji, R. Chen and Y. Xiong, Biosens. Bioelectron., 2018, 102, 9-16.

41 A. M. Smith, L. E. Marbella, K. A. Johnston, M. J. Hartmann, S. E. Crawford, L. M. Kozycz, D. S. Seferos and J. E. Millstone, Anal. Chem., 2015, 87, 2771-2778.

42 U. W. Gedde, J. Viebke, H. Leijström and M. Ifwarson, Polym. Eng. Sci., 1994, 34, 1773-1787.

43 J. R. Hulett, Q. Rev., Chem. Soc., 1964, 18, 227-242.

This journal is @) The Royal Society of Chemistry 2019

RSC Adv., 2019, 9, 36670-36679 | 36679 